\title{
Modular on-Road Self-Guided Automatic Vehicle and Concept of Self-Power Transmission
}

\author{
Akansha Varshney, Abhishek Aryan, Ashima Singh, Naveen Dubey, Anubhav Kumar Singh
}

\begin{abstract}
A self-guided automated robot is basically a robot designed to follow a line or path which is already pre-determined by the user. The main focus of this report is to design a selfguided automated path following robot which is more reliable by eliminating the demerits of the traditionally used robots by the idea of making it a magnetic field line following robot. This paper presents the principle of a magnetic field line following robot and also explains its design and working. Here, the design of a self-guided automated magnetic field line following robot using LC tank circuits and resonant coupling for navigation purposes is explained. Also this paper gives the idea of a path that can be made invisible and can be buried under the ground and also focuses on the various electrical circuitries involved in this self-guided automated magnetic field line following the robot. This paper discusses all the components that are used in this self-guided automated vehicle. The applications and the advantages of this self-guided automated magnetic field line following robot over the traditional path following robots are discussed along with the future upgradations that can be done to refine it further.
\end{abstract}

Keywords: LC, LFR, RIC, PWM, WPT

\section{INTRODUCTION}

A path following robot is basically just a robot which is designed to follow a line or a path which is predetermined by the user. As generally, path following robots are selfdriven so the path must be carefully determined by the user. This line or a path may be as simple as a physical white line on the floor or can be a complex as an embedded line, laser guide markers and magnetic markers. In order to detect these markers or lines various sensing schemes can be employed for example optical, magnetic, GPS, radar, LIDAR (light detection and ranging) [1]. In this paper we will mainly focusing on the magnetic sensing technique.

Among these path following robots, one of the most popularly used path followers is a light following robot, popularly known as LFR. LFR uses infrared sensors to detect its path [2]. But the main disadvantage with LFR is that it could not function properly in sunlight and in case when there is excessive dust on the path, so the idea was to overcome these shortcomings. So we thought of a magnetic field line follower, unlike the conventional magnetic guided robot which used to work on a magnetic tape [3].

Revised Manuscript Received on April 25, 2020.

* Correspondence Author

Akansha Varshney*, Student, B.tech, Electrical and Electronic Engineering, Galgotias College of Engineering and Technology.

Abhishek Aryan, Student, B.tech, Electrical and Electronic Engineering, Galgotias College of Engineering and Technology

Ashima Singh, Student, B.tech, Electrical and Electronic Engineering, Galgotias College of Engineering and Technology

Naveen Dubey, Student, B.tech, Electrical and Electronic Engineering, Galgotias College of Engineering and Technology

Anubhav Kumar Singh , Student, B.tech, Electrical and Electronic Engineering, Galgotias College of Engineering and Technology

(c) The Authors. Published by Blue Eyes Intelligence Engineering and Sciences Publication (BEIESP). This is an open access article under the CC BY-NC-ND license (http://creativecommons.org/licenses/by-nc-nd/4.0/)
Also unlike its preceding model which used a magnetic tape as its path, the path of our model can be made invisible from outside and wires can be laid underground. And one of the biggest advantages with our model is that it remains totally unaffected by any climatic condition exact for lightning which still can be prevented by using lightning arrestors.

Moreover the existing robots are came up with the attached battery packs for the main power supply or a fixed point charging methods but in this paper we present the idea to draw the power from the path itself by using wireless power transfer (WPT) method. Hence there will be no requirement for the battery in the robot which proved to be more efficient in the future. This paper presents the coil structure for the inductive power transfer to the vehicle by maximum flux linkage between the transmitting and receiving coils. Hence this model is self-guided as well as self-powered too. Since there is no battery is required, the model proved to be more reliable and cost efficient when compared with the conventional models.

These types of robots are commonly used in material handling facilities such as warehouses, manufacturing industries etc. Present day these types of vehicles vary from the classic ones as depicted for example in the books of Jünemann and Schmidt (2000) and Tompkins et al. (2003) in a few regards [4]. As opposed to utilizing fixed paths, numerous cutting edge robots are free-running, which implies their preferred tracks are programming modified, and can be changed generally simple when new stations or streams are included. A subsequent contrast is standing out they can be controlled. Specialist innovation permits choices to be taken by these shrewd vehicles that in the past were taken by focal controllers. This prompts versatile, selflearning vehicles and is especially proper for huge, complex frameworks with numerous vehicles and much potential vehicle obstruction. These advancements don't infer that the customary basic decision making problem become out of date. Or maybe, they lead to new difficulties for inquire about.

There are few survey papers on these robotic vehicles. Be that as it may, they focus on just restricted portions of the issue (Qiu et al. (2002) center around booking and steering issues) or are most certainly not modern (Sinriech, 1995; Co and Tanchoco, 1991; King and Wilson, 1991). Besides, they overlook a few areas, for example, idle vehicle positioning and battery management [5].

Moreover, no. of review papers has been published on the wireless charging system for these types of vehicles. These includes fixed spot charging, dynamic charging by using wireless power transfer (WPT) [6]. Various types of coil design for receiver and transmitter coils has also been proposed. 


\section{Modular on-Road Self-Guided Automatic Vehicle and Concept of Self-Power Transmission}

\section{PRINCIPLE}

An AC current flowing in a wire produces a magnetic field [7]. This field is picked up by coils on the robot. The wireless power transfer mechanism to transfer the inductive power in order to provide power supply to the system [8].

\section{A. Resonant Inductive coupling (RIC):}

RIC is the combination of both inductive coupling and resonance. Utilizing the idea of resonance it makes the two items to connect each other unequivocally. Inductance incites current in the circuit.

The loop gives the inductance. The capacitor is associated in parallel to the coil. Energy will move to and fro between magnetic field encompassing the loop and electric field around the capacitor. Here the radiation losses will be insignificant.

\section{B. Wireless power transfer (WPT):}

Wireless power transfer (WPT), remote power transmission, wireless energy transmission (WET), or electromagnetic power move is the transmission of electrical energy without wires as a physical connection [9]. In a remote power transmission framework, a transmitter device, driven by electric power from a power source, produces a period differing electromagnetic field, which transmits control crosswise over space to a receiver device, which concentrates control from the field and supplies it to an electrical load. The innovation of wireless power transmission can take out the utilization of the wires and batteries, in this manner expanding the portability, comfort, and security of an electronic gadget for all users. Wireless power move is valuable to control electrical gadgets where interconnecting wires are badly arranged, unsafe, or are unrealistic.

\section{WORKING}

Magnetic field follower based on LC tank circuit ,which is the proposed model will be comprised of an Induction wire which creates a magnetic field, In proposed model will have an inductance of $46 \mu \mathrm{H}$ and a capacitor of $0.22 \mu \mathrm{F}$ and will have power supply of variable $30 \mathrm{~V}$ power supply. $\mathrm{N}$ channel MOSFET is utilized for tuning the coil at resonating frequency of $50 \mathrm{kHz}$ sine wave.

The transmitter coil which is buried under the way which will comprise of an inductor coil creates a frequency of $50 \mathrm{kHz}$, which will be given a power supply of variable 30 $\mathrm{V}$. Signal to it will be given by the function generator which will create a frequency of $50 \mathrm{kHz}$ and to tune the power supply, MOSFET will likewise be there. There is a dc loop in the bot which will have a resonating frequency of $50 \mathrm{kHz}$ that will be the same as the frequency of the transmitter coil.

Since, there is a transmitter coil and receiver and both are in the kind of a coupled mutual induction. So the power transferred by the transmitting coil is received by the sensors of both side (one sensor on each side) of the bot. Now, based on the PWM technique and the signal which will be received by the LC tank circuit as a sensor and it will also be sent to the Arduino which is utilized as a Micro-Controller will compute the center point of the transmitting coil and will decide the zero position of path [10].

After which, motor driver L293D which is fed to the DC motor (5V $100 \mathrm{rpm}$ ) will get the signal from the MicroController along with the power supply because of which bot will just moves in alignment with the loop on its center position. Arduino utilizes both the sensors and subtract the signal by both receiver and resultant is fed as PWM signal in the motor and correspondingly it plays out the operation.

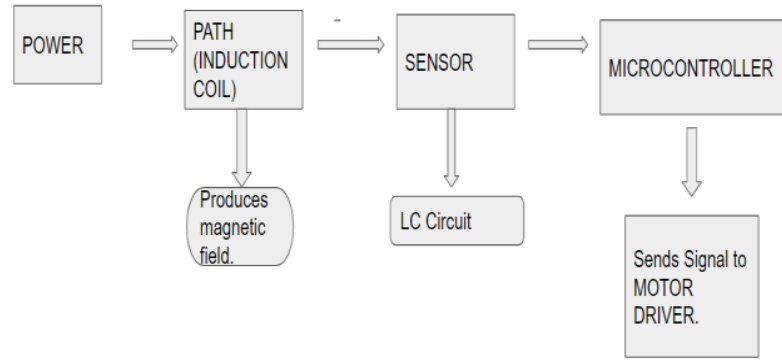

Fig - Process of Operation

\section{A. Design of Transmitter coil}

The formation of the current requires an AC generator. Its frequency is chosen high enough to be easy to pick up with small coils, but low enough to use low cost components and not to generate electro-magnetic interferences in the vicinity. A $50 \mathrm{KHz}$ frequency gave fine outcomes. A function generator creates a sine wave and an n-channel MOSFET is utilized to tune the circuit with the power supply [11]. The wire which is covered in the ground is essentially an inductance loop associated with a capacitor of about 0.22 of. The inductance of the wire is $46 \mu \mathrm{H}$. The power supply used is variable $30 \mathrm{~V}$.
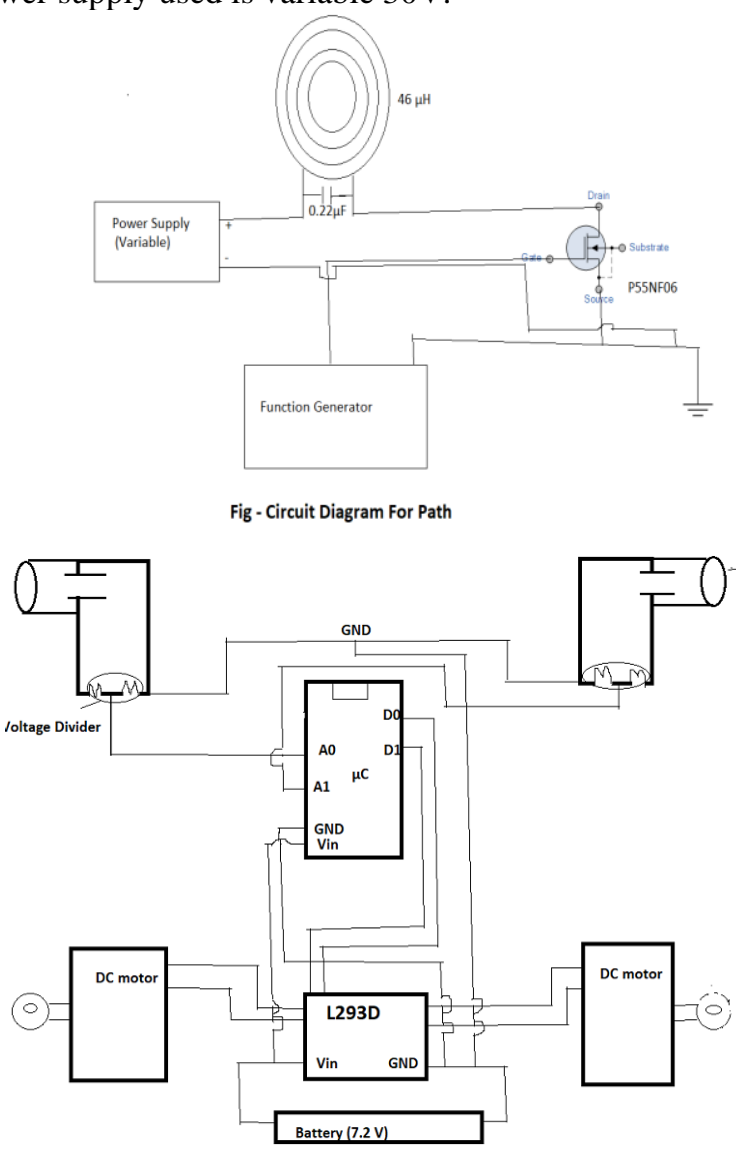

Fig - Circuit Design For Bot

Published By:

Blue Eyes Intelligence Engineering

\& Sciences Publication

(C) Copyright: All rights reserved. 


\section{B. Receiving coil:}

There are two receiving coils in the robot one is for guidance to the robot and the other coil is for the power supply to the bot.

\section{Sensor coil for navigation:}

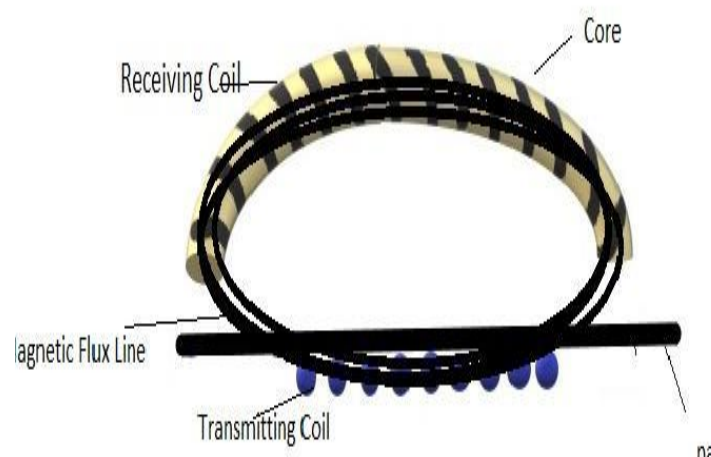

path

\section{Fig: Cross Sectional View}

Two LC tank are required to sense the magnetic field from the path. The inductance coil which receives signal from the transmitting coil have value about $10.132 \mu$ h each. The capacitor connected have capacitance value .001 $\mu \mathrm{f}$. The maximum voltage required by the microcontroller is 5 volts so to limit the voltage across the capacitor voltage divider is used .This voltage is sent to the microcontroller which drives the motors and the robot. This circuit arrangement used for the path detection and navigation.

\section{Receiving coil for the power supply:}

The robot consists of the inductive coil which is winded very densely around the ferrite core to ensure the maximum flux linkage. For the maximum flux linkage the core used is a C- shaped laminated core. This coil when linked with the transmitter coil buried in the ground, there is a change in the flux linkage which produces power in the coil.

The coil produces AC and to convert AC, rectifier and a capacitor is used to get the smooth DC waveform. This output voltage is fed to the motors.

\section{ADVANTAGES}

After Contrasted with the other line following automated vehicle which follows pre decided path, it will have following certain application which will make it more valuable and less expensive than different modes which are present in real life. Right now battery innovation is the most slacking strife issue which is growing gradually and bringing about part of intensity wastage.

In the present situation, petroleum products assets are arriving at the end because of which there might be possibilities that these could end after some time. With this model, we worked and concentration that as well and planned it so as to not require battery ,so clearly it is sparing the assets which are coming towards the end and that are significant as well. Other line following vehicle which are available now of these classifications are influenced by sunlight, dirt, so it has advantage over various line-following vehicle and preferences over that.

Most significant piece of this model is that there will be no compelling reason preferences superficially which must be trailed by it, it could likewise work if the wire will even under the surface. It will likewise have an impact framework which will be extremely useful in maintaining a distance from mishap and wounds and gainful towards society as well.

\section{RESULT}

Transmitting Coil

Table I- Transmitting coil readings

\begin{tabular}{|l|l|l|l|}
\hline $\begin{array}{l}\text { LC Tank } \\
\text { Circuit }\end{array}$ & & Power Supply & $\begin{array}{l}\text { Freque } \\
\text { ncy }\end{array}$ \\
\hline Capacitance & Inductance & & \\
\hline $0.22 \mu \mathrm{F}$ & $46 \mu \mathrm{H}$ & $30 \mathrm{~V}$ & $50 \mathrm{kHz}$ \\
\hline
\end{tabular}

Receiving Coil

Table II- Receiving coil readings

\begin{tabular}{|l|l|l|}
\hline LC Tank Circuit & & $\begin{array}{l}\text { Rating of DC } \\
\text { motor }\end{array}$ \\
\hline Capacitance & Inductance & \\
\hline $0.001 \mu \mathrm{F}$ & $10.132 \mathrm{mH}$ & $\begin{array}{l}5 \mathrm{~V}, \\
100 \mathrm{rpm}\end{array}$ \\
\hline
\end{tabular}

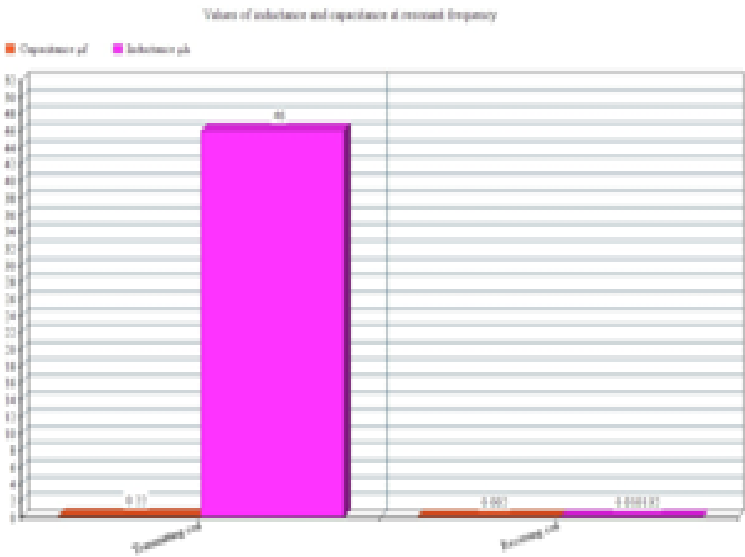

Fig. The graph showing the comparison of inductance and capacitance between transmitting and receiving coils.

The performance of the modular on-road self-guided automatic vehicle with respect to path following, power monitoring, reliability and safety was evaluated. An inductive wired track was laid out according to the intended predefined path in the real working environment. The robot ran on the desired path based on the zero position in accordance with the given resonance frequency which is chosen as $50 \mathrm{~Hz}$. The wire had an inductance of $46 \mu \mathrm{H}$, a capacitance of $0.22 \mu \mathrm{F}$ and a power supply of variable $30 \mathrm{~V}$ was given to it. Two LC tank were used to sense the magnetic field from the path. The inductance coil which receives signal from the transmitting coil have value about $10.132 \mu \mathrm{h}$ each.

Published By:

Blue Eyes Intelligence Engineering \& Sciences Publication

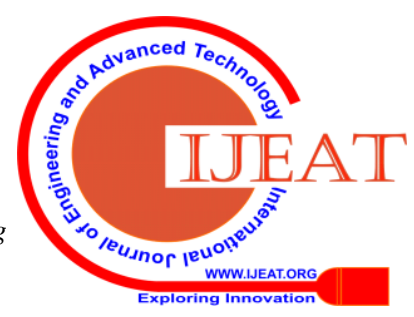




\section{Modular on-Road Self-Guided Automatic Vehicle and Concept of Self-Power Transmission}

The capacitor connected have capacitance value .001 $\mu$ f.The robot stably navigated on the desired path. Navigation was not influenced by any external conditions such as sunlight or dust particles which gives this model a major advantage over preceding models and hence makes it very reliable.

\section{CONCLUSION}

In this paper the idea of a self-powered and self-guided robot is explicitly studied. With the help of wireless power transfer we can run the vehicle without any battery. Hence the idea is efficient and reliable as it eliminates the bot can be equipped with sensors like ultrasonic sensor, infrared sensor etc. to make it smarter and advanced. The navigation depends on the strength of the magnetic field that's why it is more accurate. The path is predefined making it more useful for various applications. The running cost efficiency is better as it requires less maintenance. All the characteristics make it more reliable and robust. Due to replacement of batteries, the weight of the robot reduced by removing additional circuitry required for the battery arrangement.

\section{REFERENCES}

1. A review on Design \& navigation techniques of Automated Guided Vehicles systems (AGVs), JETIR May 2019,volume 6, issue 5.

2. https://www.elprocus.com/line-follower-robot-basics-controlling/.

3. Navigation of automated guided vehicles using magnet spot guidance method, Robotics and Computer-Integrated Manufacturing 28(3):425-436 - June 2012

4. A Review Of Design And Control Of Automated Guided Vehicle Systems,Tuan Le-Anh and M.B.M. De Koster, ERIM REPORT SERIES RESEARCH IN MANAGEMENT, May 2004.

5. Network design models for discrete material flow systems: A literature review, International Journal of Advanced Manufacturing Technology 10(4):277-291 · January 1995.

6. Dynamic Wireless Charging of Electric Vehicles En Route, Leandros A. Maglaras, ET all, IEEE, MARCH 2016.

7. https://en.wikipedia.org/wiki/Magnetic field

8. R. Bosshard, J. W. Kolar, "Inductive power transfer for electric vehicle charging: Technical challenges and tradeoffs", IEEE Power Electronics Magazine, vol. 3, no. 3, pp. 22-30, 2016.

9. Choi, S.Y.; GU, B.W.; Jeong, S.Y.; Rim, C.T. Advances in wireless power transfer systems for roadway-powered electric vehicles. IEEE J. Emerg. Sel. Top. Power Electron. 2015, 3, 18-36.

10. T. Hata and T. Ohmae, "Position detection method using induced voltage for battery charge on autonomous electric power supply systems for vehicles," in Proc. 8th IEEE Int. Workshop Adv. Motion Control, 2004, pp.187-191.

11. Lo SH, Buchanan DA, Taur et al. Quantum-mechanical modeling of electron tunneling current from the inversion layer of ultra-thin-oxide nMOSFET's. IEEE E D Lett. 1997; 18(5): 209p.

\section{AUTHORS PROFILE}

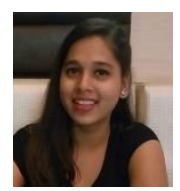

Akansha Varshney, perusing B.tech in the stream of Electrical and Electronic Engineering in Galgotias College of Engineering and Technology.

E-mail- akansha29121998@gmail.com

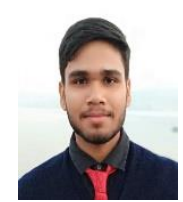

Abhishek Aryan, perusing B.tech in the stream of Electrical and Electronics Engineering in Galgotias College of Engineering and Technology.

E-mail- aryanabhisheksg@gmail.com

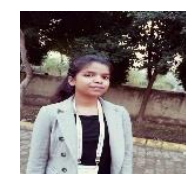

Ashima Singh, perusing B.tech in the stream of Electrical and Electronics Engineering in Galgotias College of Engineering and Technology.

E-mail- ashima23bi@gmail.com

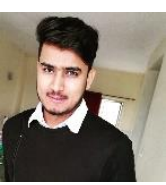

Naveen Dubey, perusing B.tech in the stream of Electrical and Electronics Engineering in Galgotias College of Engineering and Technology. E-mail- dubeynaveen99@gmail.com

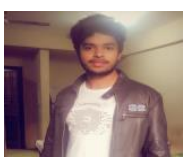

Anubhav Kumar Singh, perusing B.tech in the stream of Electrical and Electronics Engineering in Galgotias College of Engineering and Technology. E-mail- anubhav7singh7@gmail.com

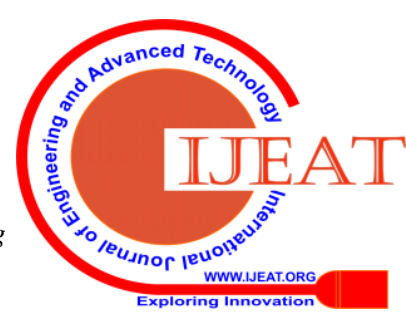

